Enhanced Sensitivity to Angry Voices in People with Features of the Broader Autism Phenotype

Valerie M. Z. Yap ${ }^{1}$, Neil M. McLachlan ${ }^{1}$, Ingrid E. Scheffer ${ }^{2,3}$, and Sarah J. Wilson ${ }^{1,{ }^{*}}$

${ }^{1}$ Melbourne School of Psychological Sciences, The University of Melbourne, Parkville, Victoria 3010, Australia, e-mail: sarahw@unimelb.edu.au, Tel: +61-3-83446378

${ }^{2}$ Departments of Medicine and Paediatrics, The University of Melbourne, Austin Health, Heidelberg, Victoria 3084, Australia

${ }^{3}$ Department of Neurology, The Royal Children's Hospital, Parkville, Victoria 3052, Australia

Funding: This research was supported in part by Award Number W81XWH-12-1-0490 from the United States Department of Defence (DoD) office of the Congressionally Directed Medical Research Programs (CDMRP)

Conflict of Interest: The authors declare that they have no conflict of interest

\title{
Abstract
}

The present study examined whether the ability to recognize vocal emotional expressions is negatively related to features of the Broader Autism Phenotype (BAP) in the general population. We assessed 61 typically developing adults on a BAP self-report measure (Broader Autism Phenotype Questionnaire) and a purpose-developed online emotion recognition task for efficient delivery of non-linguistic vocal stimuli corresponding to the six basic emotions. Contrary to expectations, we found that higher self-ratings of rigid BAP traits correlated with better recognition accuracy and higher intensity ratings for angry voices. We interpret this anger-specific association as an advantage for enhanced threat detection in the BAP and discuss this finding in the broader context of personality research and interpersonal theory.

Keywords: Broader Autism Phenotype, emotion recognition, vocal affect bursts, anger, threat sensitivity, rigidity 
Enhanced Sensitivity to Angry Voices in People with Features of the Broader Autism Phenotype

Autism Spectrum Disorder (ASD) is a pervasive neurodevelopmental condition characterized by two primary symptom domains of deficits in social communication and fixated interests or repetitive behaviours (American Psychiatric Association, 2013). Individuals with ASD are often reported to have reduced social cognition and empathy (Baron-Cohen, 2001; Baron-Cohen \& Wheelwright, 2004), which include difficulties with recognizing the emotions of others (Harms, Martin, \& Wallace, 2010; Lindner \& Rosen, 2006; Philip et al., 2010). In this study, we examined whether emotion recognition difficulties are also associated with subclinical characteristics of ASD in the general population, known as the Broader Autism Phenotype (BAP). We based our assessment on the recognition of emotional vocal expressions, particularly non-linguistic expressions that are not confounded by properties of speech.

\section{The Broader Autism Phenotype}

The BAP describes a group of behavioral and personality characteristics that are milder expressions of ASD features (Sucksmith, Roth, \& Hoekstra, 2011). Unlike ASD, however, the BAP is not considered a clinical disorder, as any functional difficulties do not cause significant impairment in daily life (Gerdts \& Bernier, 2011). BAP features have been documented in approximately $20-40 \%$ of family members of individuals with ASD, providing strong evidence for a genetic liability to the disorder (Bolton et al., 1994; Constantino, Zhang, Frazier, Abbacchi, \& Law, 2010; Gamliel, Yirmiya, Jaffe, Manor, \& Sigman, 2009; Georgiades et al., 2013; Gerdts \& Bernier, 2011; Sasson, Lam, Parlier, Daniels, \& Piven, 2013). Further, family studies have reported that the BAP is more prevalent in monozygotic (77-92\%) than dizygotic (10\%) twins (Bailey et al., 1995; Le 
Couteur et al., 1996), first-degree than more distant relatives (Pickles et al., 2000), and multiplex compared to single-incidence families (Gerdts, Bernier, Dawson, \& Estes, 2013; Losh, Childress, Lam, \& Piven, 2008; Virkud, Todd, Abbacchi, Zhang, \& Constantino, 2009).

The BAP has been examined beyond the context of heritability in ASD families, with studies reporting that milder ASD-like characteristics are also distributed within the general population (Constantino \& Todd, 2003; Hoekstra, Bartels, Verweij, \& Boomsma, 2007). Assessment of BAP features in population-based samples include self- and informant-report questionnaires, of which the Autism Spectrum Quotient (AQ; Baron-Cohen, Wheelwright, Skinner, Martin, \& Clubley, 2001), Social Responsiveness Scale (SRS; Constantino \& Gruber, 2005), and Broad Autism Phenotype Questionnaire (BAPQ; Hurley, Losh, Parlier, Reznick, \& Piven, 2007) are most common. Of note, an exploratory factor analysis of scales from these questionnaires revealed three primary dimensions of the BAP in the general population: aloofness, pragmatic language difficulties, and rigidity (Wainer, Ingersoll, \& Hopwood, 2011). The aloof dimension covers reduced social awareness or interests, while the pragmatic language dimension covers poor understanding or inappropriate means of communication within a social context. The rigid dimension covers resistance to change or reduced behavioural and cognitive flexibility. Overall, these BAP dimensions correspond to the three key domains of impairment in ASD (i.e., social interaction difficulties, communication deficits, and stereotyped repetitive interests or behaviors) defined by former DSM-IV diagnostic criteria (American Psychiatric Association, 2000).

Individuals from the general population who score higher on BAP measures may experience a range of interpersonal difficulties, including poor friendship history (i.e., reduced interest in making friends, fewer friends, shorter duration of friendships), increased loneliness, lower empathy, anxious or avoidant romantic attachment style, and higher 
frequency of being bullied (Jamil, Gragg, \& DePape, 2017; Jobe \& White, 2007; Kunihira, Senju, Dairoku, Wakabayashi, \& Hasegawa, 2006; Lamport \& Turner, 2014). Individuals with aloof traits are also more likely to form satisfying relationships with other aloof individuals (e.g., friends, college roommates), but not with warmer, more socially engaged individuals (Faso, Corretti, Ackerman, \& Sasson, 2016).

\section{Emotion Recognition in the BAP}

The ability to identify and understand the emotional cues of others is important for effective social communication, presenting an interesting but fairly novel area for BAP research in the general population. At present, research is largely confined to the study of facial expressions. Poljac, Poljac, and Wagemans (2012) reported that college students with higher AQ scores were worse at identifying facial expressions representing the basic emotions of anger, disgust, and sadness. The high-AQ scorers were also less sensitive to milder or more subtle expressions of these emotions and relied on more intense facial displays for accurate identification. Another study with college students found that higher BAPQ scores (specifically for aloofness and pragmatic language difficulties) and poorer social skills on a role-playing measure correlated with lower recognition accuracy for milder facial expressions, but not for more intense expressions (Sasson, Nowlin, \& Pinkham, 2013). Sasson et al. (2013), however, did not specify whether the correlations applied to a composite recognition score across the emotions assessed (i.e., happy, sad, angry, fearful, and neural faces) or to specific emotions. Of note, these findings are consistent with clinical studies showing reduced facial emotion recognition in individuals with ASD (Harms et al., 2010) and their family members (Kadak, Demirel, Yavuz, \& Demir, 2014; Palermo, Pasqualetti, Barbati, Intelligente, \& Rossini, 2006).

More research is warranted to examine whether BAP traits in the general population are associated with the recognition of emotions expressed through modalities other than the 
face. The voice, in particular, carries crucial information about an individual's affective state that can be discerned by a listener even when the individual is out of immediate view (Johnstone \& Scherer, 2000). Studies have found that individuals from the general population can identify emotions in speech prosody at accuracy rates well above chance (55$65 \%$ ), drawing from acoustic cues in the voice like fundamental frequency, speech rate, intensity, and voice quality (Banse \& Scherer, 1996; Johnstone \& Scherer, 2000; Scherer, 2003). Clinical research has shown that this ability is compromised in ASD (Golan, BaronCohen, Hill, \& Rutherford, 2007; Lindner \& Rosen, 2006; Philip et al., 2010; Rutherford, Baron-Cohen, \& Wheelwright, 2002), although evidence for an impairment is not always consistent, likely due to methodological differences across studies (Baker, Montgomery, \& Abrandom, 2010; Grossman, Bemis, Skwerer, \& Tager-Flusberg, 2010). It is not surprising that affected individuals may have difficulty understanding emotional cues in the voice, since other socio-emotional difficulties like theory of mind deficits (Baron-Cohen, 2001), reduced empathy (Baron-Cohen \& Wheelwright, 2004), and alexithymia (Bird \& Cook, 2013) are often reported in ASD. Research has also shown that parents of children with ASD demonstrate poorer performance on "mind reading in the voice" (theory of mind task), which included the recognition of complex emotions in speech prosody (Tajmirriyahi, Nejati, Pouretemad, \& Sepehr, 2013).

Using a sample of college students, Ingersoll (2010) examined whether BAP features in the general population are more broadly associated with "nonverbal sensitivity" across modalities of emotional expressions, including faces, body postures, and speech prosody. Using the AQ, they found that self-ratings of poorer social skills and greater attention to detail/patterns correlated with poorer recognition of emotional faces, specifically sadness and anger (but not happiness and fear). In contrast, AQ scores did not correlate with the recognition of specific emotions in body postures and speech prosody. These results suggest 
that poor emotion recognition ability associated with BAP traits in the general population is not generalized across modalities, but specific to facial expressions. However, Ingersoll (2010) suggested that correlations were found exclusively for the recognition of facial emotions because the task was easy for most participants and significantly simpler than the recognition of postures and speech prosody.

It is worth considering whether the use of alternate BAP measures and emotional stimuli would yield findings that support a link between BAP traits and vocal emotion recognition ability in the general population. While Ingersoll (2010) used the AQ to measure BAP traits in her sample, a more recent study found that the BAPQ has better psychometric properties for assessing the BAP in the general population (Ingersoll, Hopwood, Wainer, \& Donnellan, 2011). Compared to the AQ, the BAPQ has higher internal consistency, higher criterion validity (to outcome measures of mood/anxiety symptoms and personality traits), and a replicable factor structure corresponding to the three theoretical dimensions of the BAP (i.e., aloofness, pragmatic language difficulties, rigidity; Ingersoll et al., 2011). Further, the BAPQ was originally developed to determine milder characteristics of the BAP in parents of children with ASD (Hurley et al., 2007), unlike the AQ which was developed to identify high-functioning individuals with a clinical diagnosis of ASD (Baron-Cohen \& Wheelwright, 2004).

As for vocal stimuli, although most emotion recognition studies (within and outside the ASD context) have employed speech prosody, valuable information about an individual's affective state is also communicated through non-linguistic vocalizations or vocal "affect bursts" (Belin, Fillion-Bilodeau, \& Gosselin, 2008; Hawk, van Kleef, Fischer, \& van der Schalk, 2009; Schröder, 2003; Simon-Thomas, Keltner, Sauter, Sinicropi-Yao, \& Abramson, 2009). Vocal affect bursts include laughter, screams, cries, sighs, and groans. Compared to speech prosody, affect bursts are considered to be less culturally dependent, more raw or 
reflexive, and hence, more accurate representations of affective states that are not confounded by linguistic content (Belin et al., 2008; Hawk et al., 2009). They are generally easier to recognize, with accuracy rates ranging from 68 - 81\% (Belin et al., 2008; Schröder, 2003). They also have evolutionary significance, with some occurring early in development before language and speech are acquired (e.g., infants crying to communicate with their mother; Zeifman, 2001).

\section{Present Study}

As yet, research has not been conducted to assess whether poorer recognition of vocal affect bursts constitute a marker of the BAP in the general population. This is significant if we are to accurately understand the full spectrum of performance in milder phenotypes of ASD. We examined correlations between self-ratings on the BAPQ and recognition accuracy for vocal affect bursts representing the six basic emotions (i.e., anger, disgust, fear, happiness, sadness, and surprise) in a non-clinical sample who have no family members with ASD. In addition, we assessed whether levels of BAP traits were associated with intensity ratings of vocal affect bursts (i.e., how "strong" participants perceived the emotions to be). Intensity is a common dimension of physiological arousal in emotion research (Juslin \& Laukka, 2001; Laukka, Juslin, \& Bresin, 2005) and served as an additional measure of sensitivity to emotional stimuli in our study.

Based on prior research on social difficulties in ASD and the BAP, we hypothesized that higher scores on each BAPQ scale measuring the three primary BAP dimensions (i.e., aloofness, pragmatic language difficulties, and rigidity) would correlate with lower recognition accuracy and lower intensity ratings for vocal affect bursts from each basic emotion category. To test these hypotheses, we developed a new online program for assessing recognition and intensity ratings for a selection of validated stimuli from the Montreal Affective Voices (MAV) battery of vocal affect bursts (Belin et al., 2008). 


\section{Method}

This project was approved by the Human Ethics Advisory Group at The University of Melbourne.

\section{Participants}

Sixty-three fluent English speakers (35 females) were recruited from the community by word-of-mouth and advertisements. This sample size was determined to achieve $81 \%$ power at an alpha level of .05 for correlation coefficients of at least moderate effect size $(r>$ .35) using a web-based sample size calculator (StatsToDo; Chang, 2014). One male participant was excluded from the analysis due to malfunctioning of the computer software at the time of testing. Eight participants reported being previously diagnosed with a psychiatric condition ( $n=4$ with depression, $n=3$ with anxiety, $n=1$ with comorbid depression and anxiety), of which only three reported ongoing symptoms. No significant mean differences were found across all performance measures between participants with and without a psychiatric history. However, the male participant with comorbid depression and anxiety was excluded from the analysis, as he was an outlier in our performance measure of vocal emotion recognition (i.e., scored below three standard deviations of the mean on recognition accuracy for angry and sad voices). Characteristics of the 61 remaining participants are shown in Table 1. No participant reported having ASD or family members diagnosed with ASD.

[Insert Table 1 about here]

\section{Materials}

Montreal Affective Voices (MAV). The MAV is a validated set of non-linguistic vocal affect bursts designed for cross-cultural research on auditory emotional processing (Belin et al., 2008). MAV stimuli were recorded by 10 different actors (five females) under instruction to produce short emotional interjections across a range of emotion categories 
using the vowel ah (/a/). The original set of MAV stimuli consists of 90 vocalizations corresponding to eight emotion categories of anger, disgust, fear, happiness, sadness, surprise, pleasure, pain, and a neutral category (no emotion). To minimize task duration, we selected a subset of 42 vocalizations that corresponded to the six basic emotions (anger, disgust, fear, sadness, surprise, happiness) and the neutral category, with three male and three female vocalizations per category. For each category, we refined our selection to vocalizations that sounded the most similar based on consensus judgements between authors (e.g., angry voices with a distinguishable growl and roughness, disgusted voices with a distinguishable rise in pitch contour corresponding to the interjection "eugh").

Emotion Recognition Task. The Emotion Recognition Task uses novel online software that we purposely designed for efficient and randomized delivery of MAV stimuli (Anonymous, 2013; reference removed from bibliography for blind manuscript). The program includes a purpose-built graphic interface that allows participants to hear each stimulus as often as required before rating it in terms of its emotion category and degree of intensity (Figure 1). Correct classification of emotions involved a seven-alternative forced choice task (six basic emotions and neutral category), and intensity ratings were made on a 7point Likert-type scale $(1=$ weak emotion to $7=$ strong emotion $)$. An intensity rating of 0 was expected for trials of the neutral category. There were 42 trials in total, corresponding to a maximum total emotion recognition accuracy score of 42 points. Responses were stored in an XML data file located on a secure online server and downloaded for subsequent analyses.

[Insert Figure 1 about here]

Broad Autism Phenotype Questionnaire (BAPQ). The BAPQ is a 36-item selfreport questionnaire designed to measure features of the BAP that correspond with milder impairments of ASD (Hurley et al., 2007). The BAPQ has three 12-item scales: (a) Aloof Personality (e.g., "I prefer to be alone rather than with others"), (b) Pragmatic Language (e.g., 
"I can tell when someone is not interested in what I am saying"), and (c) Rigid (e.g., "I have a hard time dealing with changes in my routine"). All scales have high internal reliability, with alpha coefficients ranging from .85 to .94 (Hurley et al., 2007). Participants rate how often each item applies to them on a 6 -point Likert-type scale $(1=$ very rarely to $6=$ very often $)$.

\section{Procedure}

All participants provided signed informed consent. The emotion recognition task and BAPQ were presented in a counter-balanced order across participants. Participants completed the emotion recognition task on a computer in a soundproof room at The University with Melbourne, with stimuli presented through an external speaker adjusted to a comfortable listening volume. Participants also completed a brief health screen about their medical and psychiatric history, and an assessment of general intellectual functioning using the Wechsler Abbreviated Scale of Intelligence (WASI; Wechsler, 1999).

\section{Results}

Data were analysed using IBM SPSS Statistics (Version 21.0). The distribution of each variable was assessed for normality according to z-scores of the skewness value $(z<$ 2.58). Variables that were not normally distributed were subjected to the $\log 10$ or arcsine transformation (Sokal \& Rohlf, 1995). All transformed data were subsequently checked for normality. For ease of interpretation, parametric tests on the untransformed (original) data are reported whenever the transformed (normalized) data yielded similar results. Parametric tests of the original data are also reported in place of non-parametric tests (performed on original data that remained skewed even after transformation) when results were similar across both analyses.

\section{Descriptive Statistics}

Emotion recognition accuracy. The mean total recognition accuracy across all seven categories of the Emotion Recognition Task was $81.36 \%(S D=7.03)$. Figure 2 shows 
the recognition accuracy for each basic emotion category. Overall, our findings compare favorably with those from Belin, Fillion-Bilodeau, and Gosselin's (2008) original study on the validation of MAV stimuli, with the clear exception of happiness which showed the highest accuracy scores in our study (98\%; ceiling effect) but lowest in Belin et al.'s study (60\%). The lower recognition accuracy for happiness reported by Belin et al. can be attributed to its confusion with the category of "pleasure", which was included as a forcedchoice alternative. Accordingly, Belin et al. suggested that pleasure be excluded as a response option in future MAV-related studies, as we have done here. Similarities between recognition accuracy rates from our study and Belin et al.'s (2008) suggest that our purposebuilt online task and abbreviated choice of MAV stimuli are valid for research on vocal emotion recognition.

\section{[Insert Figure 2 about here]}

In our study, voices of fear and surprise were most often confused, with approximately $20 \%$ of fearful voices mistaken to represent surprise and vice versa. Confusion between fear and surprise was also reported by Belin et al. (2008) as well as others studying vocal emotion recognition (Pell, Paulmann, Dara, Alasseri, \& Kotz, 2008; SimonThomas et al., 2009). Voices representing the six basic emotions were rarely confused with the neutral category ( $1 \%$ of all trials), indicating that participants did not show a response bias for the central response option on our graphical interface (Figure 1), but rather, actively engaged in the task. Two participants reported post-hoc that they did not know the neutral category was a response option, and we therefore excluded them from all analyses involving the neutral category (including analyses of total recognition accuracy).

Belin et al. (2008) reported gender effects for the recognition of MAV stimuli, with females performing better than males. In our study, a two-way mixed ANOVA revealed no interaction between gender and emotion category for accuracy, $F(3.33,196.53)=0.65, p=$ 
$.60, \eta_{p}{ }^{2}=0.01$, indicating that recognition ability across the six emotion categories was not significantly different for males and females (see Online Resource 1 for gender-specific means). There was also no main effect of gender on recognition accuracy, $F(1,59)=2.20, p$ $=0.14, \eta_{p}{ }^{2}=0.04$. However, there was a main effect of emotion category, $F(3.33,196.53)=$ 28.73, $p<.001, \eta_{p}{ }^{2}=0.33$. Bonferroni-corrected post-hoc analyses indicated that participants were more accurate at identifying happy and sad voices compared to other emotions ( $p<.001, d=0.92$ to 1.84 ), although accuracy was lower for sad compared to happy voices $(p<.001, d=0.81)$. No significant differences in accuracy were found among voices of disgust, anger, fear, and surprise ( $p=.78$ to 1.00$)$.

Emotion intensity ratings. The mean total intensity rating across all six basic emotion categories of the Emotion Recognition Task was $137.42(S D=25.20)$, out of a possible maximum total of 252 points. Figure 3 shows intensity ratings for each basic emotion category. A two-way mixed ANOVA revealed no interaction between gender and emotion category on intensity ratings, $F(4.53,267.09)=0.38, p=.85, \eta_{p}{ }^{2}=0.01$ (see Online Resource 1 for gender-specific means). We also found no main effect of gender on intensity ratings, $F(1,59)=0.06, p=0.82, \eta_{p}{ }^{2}=0.001$. However, we found a main effect of emotion category, $F(3.33,196.53)=28.73, p<.001, \eta_{p}{ }^{2}=0.33$. Bonferroni-corrected post-hoc analyses indicated that participants rated voices of happiness and sadness to be stronger than all other emotions ( $p<.001, d=0.82$ to 2.30 ), whereas voices of surprise were rated to be of the weakest intensity ( $p<.001, d=0.96$ to 2.30 ).

[Insert Figure 3 about here]

BAPQ. The mean total BAPQ score was 2.52 points $(S D=0.52)$. Descriptive statistics for self-ratings of the three primary BAP components are reported in Figure 4 (see Online Resource 2 for a distribution of scores per BAPQ scale). Scores on the BAPQ are similar to those reported in other studies assessing the general population (Ingersoll et al., 
2011; Sasson, Lam, Childress, et al., 2013), with the exception of Rigid scores, which are lower in our study. Research on the general population has also shown that females have lower scores on the BAPQ, particularly on the Aloof and Pragmatic Language scales (Ingersoll, 2010). However, a two-way mixed ANOVA revealed no interaction effect between gender and scores on BAPQ scales, $F(2,118)=1.20, p=.30, \eta_{p}{ }^{2}=0.02$ (see Online Resource 1 for gender-specific means). Further, there were no main effects of gender, $F(1$, 59) $=0.37, p=0.55, \eta_{p}{ }^{2}=0.01$, and scale, $F(2,118)=1.85, p=1.62, \eta_{p}{ }^{2}=0.03$, on BAPQ scores.

[Insert Figure 4 about here]

\section{Correlational Analyses}

Emotion recognition accuracy and the BAP. Bivariate correlations between emotion recognition accuracy and self-ratings of BAP features are shown in Table 2. We found that better recognition of angry voices was associated with higher scores on the Rigid scale of the BAPQ, $r(59)=.32, p=.013$, with a medium effect size. This finding is counter to our hypothesis that participants with higher levels of BAP traits would be poorer at recognizing vocal emotions. No significant correlations were found for the remaining emotion categories and BAPQ scales (including BAPQ total score), suggesting specific effects of rigid BAP traits on the recognition of angry voices.

[Insert Table 2 about here]

Emotion intensity ratings and the BAP. Bivariate correlations between emotion intensity ratings and self-ratings of BAP features are shown in Table 3. We found a correlation with small effect size between higher intensity ratings for anger and higher BAPQ Rigid scores, $r(59)=.29, p=.024$. No significant correlations were found for the remaining emotion categories and BAPQ scales (including BAPQ total score). This finding is 
consistent with the specific association found between better recognition of angry voices and higher rigid BAP traits.

[Insert Table 3 about here]

Emotion recognition accuracy and intensity ratings. We found positive bivariate correlations between recognition accuracy and intensity ratings for each emotion category, suggesting that the ability to identify vocal emotions was associated with how intense the emotions were perceived to be, as also reported by other researchers (Juslin \& Laukka, 2001). Specifically, accuracy-intensity correlations with strong effect sizes were found for surprise, disgust, anger, and fear, $r(59)=.74$ to $.91, p<.001$, while correlations of medium effect sizes were found for happiness and sadness, $r(59)=.35, p<.001$.

\section{Discussion}

We examined whether self-ratings of BAP features in the general population are associated with recognition accuracy for non-linguistic vocal expressions representing the six basic emotions. We hypothesized that higher self-ratings on the three primary BAP dimensions would be associated with lower recognition accuracy of vocal affect bursts. Contrary to expectations, correlational analyses revealed that higher self-ratings of rigid personality traits were associated with better recognition of angry voices. Higher self-ratings of rigidity were also associated with higher intensity ratings for angry voices, suggesting a robust association between rigid features of the BAP and sensitivity to anger. Of note, no significant correlations (positive or negative) were found between other BAP features and recognition accuracy or intensity ratings for any of the other basic emotions.

\section{BAP Features Do Not Impair the Recognition of Vocal Affect Bursts}

Our findings suggest that individuals from the general population with more BAP features are not at a disadvantage for understanding vocal affect bursts. As such, difficulties with vocal affect burst recognition do not constitute a marker of the BAP, at least in people 
who have no family members with ASD. These findings were unexpected in light of clinical studies reporting reduced emotion recognition ability across modalities in ASD (Harms et al., 2010; Lindner \& Rosen, 2006; Philip et al., 2010). It is worth noting that some clinical studies have shown that individuals with ASD can recognize basic emotions in speech prosody as accurately as controls (Baker et al., 2010; Grossman et al., 2010), but are worse at recognizing more complex vocal emotions related to theory of mind processes (Golan et al., 2007; Rutherford et al., 2002). In a similar vein, perhaps the lack of significant negative correlations between BAP traits and vocal emotion recognition in our study reflects our use of non-linguistic stimuli (Belin et al., 2008), which resemble prototypical expressions of emotions that are generally easy to recognize and do not require much cognitive interpretation.

\section{Enhanced Sensitivity to Angry Voices in the BAP}

Using our vocal emotion recognition paradigm, we unexpectedly found positive correlations between anger-specific processing and rigid features of the BAP in the general population. Since anger is a negative-valence emotion associated with appraisals of dominance and threat, our key findings suggest that individuals who have more rigid BAP traits are particularly sensitive to threatening information in the voice. It is interesting that we found no correlations between BAP traits and the recognition of fear, another threatrelated emotion. Anger and fear can be distinguished according to their motivational tendencies, whereby anger elicits approach-related behaviour while fear evokes avoidancerelated behaviour (Carver \& Harmon-Jones, 2009). Angry signals often arise from the source of threat itself and can be used to assert social dominance, whereas fearful signals often reflect an awareness of threat which may result in submission or the goal to protect oneself (Bossuyt, Moors, \& De Houwer, 2014). 
The accurate identification of angry expressions appears to have an evolutionary advantage, as indicated by facial emotion research on the "anger superiority effect", where angry faces are recognized more quickly and accurately than happy or friendly (nonthreatening) faces when both are presented in visual search tasks (Hansen \& Hansen, 1988; Öhman, Lundqvist, \& Esteves, 2001). The anger superiority effect has also been reported in high-functioning ASD (Ashwin, Wheelwright, \& Baron-Cohen, 2006; Isomura, Ogawa, Yamada, \& Shibasaki, 2014; Rosset et al., 2011), implying that individuals with atypical social development have intact mechanisms for detecting threat, although they may rely on different strategies to do so (e.g., feature-based versus holistic processing of angry faces).

While behavioural paradigms assessing the anger superiority effect have not been adapted for auditory emotion research, an attentional bias towards angry voices can be inferred from neuroimaging studies in the general population. Specifically, studies have found enhanced activity to angry prosody in voice-selective areas (e.g., superior temporal sulcus) and regions involved with reflexive processing of salient stimuli (e.g., the amygdala), even when task demands do not require selective attention to the voice (Grandjean, 2005; Sander et al., 2005). However, studies have yet to examine whether individuals with ASD have similar neural responses to angry voices that allow them to process threatening information more efficiently.

\section{Personality, Interpersonal Styles, and Threat Detection in the BAP}

Support for sensitivity to threat in individuals with higher levels of rigid BAP traits may be drawn from the broader context of personality research. The "Big-Five" (McCrae \& John, 1992) personality trait of neuroticism has been linked with rigidity of thought and behaviour on the AQ and BAPQ (Austin, 2005; Wainer et al., 2011; Wakabayashi, BaronCohen, \& Wheelwright, 2006). Of note, neuroticism is associated with psychological distress, high trait anxiety, attentional bias to unpleasant emotional information and a greater 
tendency to appraise daily experiences as being negative (Costa \& McCrae, 1980; Gomez, Gomez, \& Cooper, 2002; Tong, 2010; Watson \& Clark, 1984). Individuals with higher levels of neuroticism and trait anxiety are also more prone to interpret ambiguous stimuli or situations as being threatening and avoid such encounters (Bar-Haim, Lamy, Pergamin, Bakermans-Kranenburg, \& van IJzenboorn, 2007; Calvo \& Castillo, 2001; Lommen, Engelhard, \& van den Hout, 2010). In addition to neuroticism, higher self-ratings of rigid traits on the BAPQ also correlate with higher rates of anxiety and obsessive-compulsiveness in the general population (Wainer et al., 2011). It is therefore possible that underlying interactions between neuroticism and anxiety in our sample may at least partially account for the anger-specific association with rigid BAP features found here.

Although rigidity is not typically discussed in terms of its social relevance, its relationship with enhanced sensitivity to threat may have consequences for social functioning in the BAP. According to interpersonal theory, successful relationships depend on the extent to which individuals are able to engage in complementary social behaviour along two dimensions of dominance/submission and affiliation/hostility (Carson, 1969; Kiesler, 1983; Leary, 1957). Complementary interpersonal styles are considered to be opposite on dominance but similar on affiliation, whereby for example, dominant behaviour is likely to elicit a submissive response (and vice versa), while friendliness or hostility is likely to elicit a warm or cold response, respectively (Kiesler, 1983).

Rigid individuals may have more difficulty adapting their behaviour to fit a variety of social contexts, as their resistance to change may prevent them from considering the perspectives of others and responding appropriately to less familiar interactions (Kiesler, 1983; Leary, 1957; Tracey, 2005). In line with increased threat sensitivity and neuroticism, it is possible that rigid individuals are more vigilant towards others who engage in interpersonal styles that do not complement their own. For example, dominant individuals who display a 
narrow range of social behaviours may feel threatened around other self-assured individuals and develop a more authoritarian approach to assert their views (e.g., Schultz, Stone, \& Christie, 1997), even if compliant or agreeable behaviour is more appropriate for the interaction. Future research is needed to determine the extent to which rigidity and sensitivity to threat contribute to lower quality of relationships and maladaptive attachment styles associated with the BAP (e.g., Jamil et al., 2017; Jobe \& White, 2007; Lamport \& Turner, 2014).

\section{Comparison with Other BAP Studies on Emotion Recognition}

Overall, our findings differ from those reported by Ingersoll (2010), who examined the association between emotion recognition across modalities and BAP features in the general population. Specifically, Ingersoll did not find a significant correlation between the recognition of angry prosody (or any other vocal emotion) and BAP features.

Methodological differences could at least partially account for this discrepancy. Ingersoll (2010) used linguistic stimuli spoken in different emotional tones (e.g., "I'm going out of the room now and I'll be back later"), which could have been confounded due to possible interactions between the semantic and affective components in speech. Ingersoll also used the AQ to measure the BAP, whereas we used the BAPQ, which has better psychometric properties and a factor structure that corresponds well with the theoretical domains of the BAP (Ingersoll et al., 2011).

It is interesting to note that a correlation between higher self-ratings of BAP traits and poorer recognition of angry faces was found in Ingersoll's (2010) study. Other researchers have also reported a negative correlation between BAP traits and facial anger recognition in the general population, particularly for less intense expressions (Poljac et al., 2012). Our proposal of enhanced threat detection in the BAP may be specific to the auditory domain. This may reflect that anger cues in the voice are more arousing than anger cues in the face, 
and hence, serve as stronger signals of dominance or impending danger. Of note, research has shown that vocal affect bursts for anger are recognized more easily than their facial equivalents (Hawk et al., 2009). The ability to detect angry voices may have a greater evolutionary advantage over angry faces, as it can alert the listener to the direction of an approaching threat even when the source of danger is out of view. Developmental studies have shown that infants can detect vocal emotions (and discriminate between positive and negative ones) earlier than facial emotions, likely due to earlier prenatal development of the auditory versus visual system (Caron, Caron, \& MacLean, 1988; Fernald, 1988; WalkerAndrews, 1997). More research is needed to examine why individuals with higher levels of the BAP are sensitive to angry voices but not faces. Future studies using cross-modal paradigms to assess anger recognition in the BAP is also recommended.

\section{Limitations}

There are a number of limitations related to task design and the demographics of our sample. The MAV stimuli (Belin et al., 2008) were recorded by professional actors upon prompting and were therefore not elicited spontaneously. Some may therefore argue that our stimuli were only prototypes of emotional expressions, not portrayals of genuine affective states, which may affect the ecological validity of our study. For example, actor-portrayed stimuli may represent stronger affective states (e.g., higher intensity), as opposed to natural expressions of the same emotion. However, the use of actor portrayals has been a primary approach in emotion research because of the ethical considerations and practical difficulties associated with eliciting genuine emotional states in natural or controlled settings (Johnstone \& Scherer, 2000). Prototypes of emotional expressions are also appropriate for such studies because they often represent display rules, or social norms that dictate appropriate expression of emotions, which must be understood for effective communication (Scherer, 2003). Further, our data showed a good distribution of intensity ratings across stimuli, suggesting 
variation in the strength of portrayed emotional states whereby not all stimuli were perceived to have high intensity.

On our computerized emotion recognition task, we did not control the number of times that participants were allowed to listen to each vocal stimulus, nor did we assess reaction time for correctly identified emotions. The latter may show greater sensitivity to individual differences in vocal emotion recognition. Despite these limitations, our accuracy rates were similar to those of Belin et al.'s (2008), indicating that our computerized task was reliable and robust for identifying differences in performance across the basic emotion categories. Further, our paradigm yielded positive correlations between recognition accuracy and intensity ratings across emotions, consistent with previous research on the role of emotion intensity in vocal emotion recognition (Juslin \& Laukka, 2001).

Our sample consisted of highly educated participants (the majority having completed, or currently completing, a postgraduate degree) whose mean IQ score fell in the High Average range. Therefore, our sample does not represent a wide distribution of intellectual functioning and educational background as expected in the general population. Despite this, our participants were from various cultural backgrounds, which may support the use of MAV stimuli in cross-cultural studies on auditory emotion processing. It is also worth noting that we did not find any significant gender differences across our emotion and BAP measures, suggesting that gender-related differences were unlikely to account for the anger-specific relationship with BAP features. A final point is that we did not formally assess mood symptoms (e.g., anxiety, depression). However, we asked participants to indicate whether they had a psychiatric history and accounted for any confounding effect of mental disorder on recognition accuracy by removing a participant (with comorbid depression and anxiety) from analysis since he was an outlier on the task.

\section{Conclusion}


In summary, this study provides new information about how BAP traits in the general population are related to vocal emotion recognition ability. We used a novel online paradigm to assess vocal recognition and intensity ratings for a selection of non-linguistic vocal affect bursts from the MAV battery. We found that the BAP dimension of rigidity was associated with enhanced recognition and higher intensity ratings specifically for angry voices. Our findings suggest that typically developing individuals with more rigid BAP traits are more sensitive to threatening stimuli within the auditory domain. Further research is warranted to understand the mechanisms underlying anger-specific associations in ASD and the BAP. 


\section{Compliance with Ethical Standards}

Disclose of Potential Conflicts of Interest: The authors declare that they have no conflict of interest.

Funding: This research was supported in part by Award Number W81XWH-12-1-0490 from the United States Department of Defence (DoD) office of the Congressionally Directed Medical Research Programs (CDMRP).

Ethical Approval: All procedures performed in studies involving human participants were in accordance with the ethical standards of the institutional and/or national research committee and with the 1964 Helsinki declaration and its later amendments or comparable ethical standards. This article does not contain any studies with animals performed by any of the authors.

Informed Consent: Informed consent was obtained from all individual participants included in the study. 


\section{References}

American Psychiatric Association. (2000). Diagnostic and statistical manual of mental disorders (4th ed.). Washington, DC: Author.

American Psychiatric Association. (2013). Diagnostic and statistical manual of mental disorders (5th ed.). Washington, DC: Author.

Ashwin, C., Wheelwright, S., \& Baron-Cohen, S. (2006). Finding a face in the crowd: Testing the anger superiority effect in Asperger Syndrome. Brain and Cognition, 61, 78-95.

Austin, E. J. (2005). Personality correlates of the broader autism phenotype as assessed by the Autism Spectrum Quotient (AQ). Personality and Individual Differences, 38, 451460. doi:10.1016/j.paid.2004.04.022

Bailey, A., Le Couteur, A., Gottesman, I., Bolton, P., Simonoff, E., Yuzda, E., \& Rutter, M. (1995). Autism as a strongly genetic disorder: Evidence from a British twin study. Psychological Medicine, 25(1), 63-77. doi:10.1017/S0033291700028099

Baker, K. F., Montgomery, A. A., \& Abrandom, R. (2010). Brief report: Perception and lateralization of spoken emotion by youths with high-functioning forms of autism. Journal of Autism and Developmental Disorders, 40, 123. doi:10.1007/s10803-0090841-1

Banse, R., \& Scherer, K. R. (1996). Acoustic profiles in vocal emotion expression. Journal of Personality and Social Psychology, 70(3), 614-636. doi:10.1037/0022-3514.70.3.614

Bar-Haim, Y., Lamy, D., Pergamin, L., Bakermans-Kranenburg, M. J., \& van IJzenboorn, M. H. (2007). Threat-Related Attentional Bias in Anxious and Nonanxious Individuals: A Meta-Analytic Study. Psychological Bulletin, 133(1), 1-24. doi:10.1037/00332909.133.1.1 
Baron-Cohen, S. (2001). Theory of mind and autism: A review. International review of research in mental retardation, 23, 169-184. doi:10.1016/S0074-7750(00)80010-5

Baron-Cohen, S., \& Wheelwright, S. (2004). The Empathy Quotient: An Investigation of adults with Aspergers syndrome or high functioning autism, and normal sex differences. Journal of Autism and Developmental Disorders, 34(2), 163-175. doi:10.1023/B:JADD.0000022607.19833.00

Baron-Cohen, S., Wheelwright, S., Skinner, R., Martin, J., \& Clubley, E. (2001). The Autism-Spectrum Quotient (AQ): Evidence from Asperger syndrome/highfunctioning autism, males and females, scientists and mathematicians. Journal of Autism and Developmental Disorders, 31(1), 5-17. doi:10.1023/A:1005653411471

Belin, P., Fillion-Bilodeau, S., \& Gosselin, F. (2008). The Montreal Affective Voices: A validated set of nonverbal affect bursts for research on auditory affective processing. Behavior Research Methods, 40(2), 531-539. doi:10.3758/BRM.40.2.531

Bird, G., \& Cook, R. (2013). Mixed emotions: The contribution of alexithymia to the emotional symptoms of autism. Translational Psychiatry, 3(7), e285. doi:10.1038/tp.2013.61

Bolton, P., Macdonald, H., Pickles, A., Rios, P., Goode, S., Crowson, M., . . Rutter, M. (1994). A case-control family history study of autism. Journal of Child Psychology and Psychiatry, 35(5), 877-900. doi:10.1111/j.1469-7610.1994.tb02300.x

Bossuyt, E., Moors, A., \& De Houwer, J. (2014). On angry approach and fearful avoidance: The goal-dependent nature of emotional approach and avoidance tendencies. Journal of Experimental Social Psychology, 50, 118-124. doi:10.1016/j.jesp.2013.09.009

Calvo, M. G., \& Castillo, M. D. (2001). Selective interpretation in anxiety: Uncertainty for threatening events. Cognition and Emotion, 15, 299-320. doi:/doi.org/10.1080/02699930126040 
Caron, A. J., Caron, R. F., \& MacLean, D. J. (1988). Infant Discrimination of Naturalistic Emotional Expressionsa: The Role of Face and Voice. Child Development, 59(3), 604-616. doi:10.2307/1130560

Carson, R. C. (1969). Interaction concepts of personality. Chicago: Aldine.

Carver, C., \& Harmon-Jones, E. (2009). Anger is an approach-related affect: Evidence and implications. Psychological Bulletin, 135(2), 183-204. doi:10.1037/a0013965

Chang, A. (2014). StatsToDo: Sample size for Pearson's correlation coefficient program. Retrieved from https://www.statstodo.com/SSizCorr_Pgm.php

Constantino, J., \& Gruber, C. (2005). Social Responsiveness Scale. Los Angeles, CA: Western Psychological Services.

Constantino, J. N., \& Todd, R. D. (2003). Autistic traits in the general population. Archives of General Psychiatry, 60, 525-530. doi: 10.1023/A:1025014929212

Constantino, J. N., Zhang, Y., Frazier, T., Abbacchi, A. M., \& Law, P. (2010). Sibling recurrence and the genetic epidemiology of autism. American Journal of Psychiatry, 167(11), 1349-1356. doi:10.1176/appi.ajp.2010.09101470

Costa, P. T., \& McCrae, R. R. (1980). Influence of extraversion and neuroticism on subjective well-being: Happy and

unhappy people. Journal of Personality and Social Psychology, 38, 668-678. doi:10.1037/0022-3514.38.4.668

Faso, D. J., Corretti, C. A., Ackerman, R. B., \& Sasson, N. J. (2016). The broad autism phenotype predicts relationship outcomes in newly formed college roommates. Autism, 20(4), 412-424.

Fernald, A. (1988). Human maternal vocalizations to infants as biologically relevant signals: an evolutionary perspective. In J. H. Barkow, L. Cosmides, \& J. Tooby (Eds.), The 
adapted mind: Evolutionary psychology and the generation of culture (pp. 391-428). Oxford: Oxford University Press.

Gamliel, I., Yirmiya, N., Jaffe, D. H., Manor, O., \& Sigman, M. (2009). Developmental trajectories in siblings of children with autism: Cognition and language from 4 months to 7 years. Journal of Autism and Developmental Disorders, 39(8), 11311144. doi:10.1007/s10803-009-0727-2

Georgiades, S., Szatmari, P., Zwaigenbaum, L., Bryson, S., Brian, J., Roberts, W., . . Garon, N. (2013). A prospective study of autistic-like traits in unaffected siblings of probands with autism spectrum disorder. JAMA Psychiatry, 70(1), 42-48. doi:10.1001/2013.jamapsychiatry.1

Gerdts, J., \& Bernier, R. (2011). The broader autism phenotype and its implications on the etiology and treatment of autism spectrum disorders. Autism Research and Treatment, 2011, 1-19. doi:10.1155/2011/545901

Gerdts, J., Bernier, R., Dawson, G., \& Estes, A. (2013). The broader autism phenotype in simplex and multiplex families. Journal of Autism and Developmental Disorders, 43, 1597-1605.

Golan, O., Baron-Cohen, S., Hill, J. J., \& Rutherford, M. D. (2007). The "Reading the Mind in the Voice" Test-Revised: A study of complex emotion recognition in adults with and without autism spectrum conditions. Journal of Autism and Developmental Disorders, 37(6), 1096-1106. doi:10.1007/s10803-006-0252-5

Gomez, R., Gomez, A., \& Cooper, A. (2002). Neuroticism and extraversion as predictors of negative and positive emotional information processing: Comparing Eysenck's, Gray's, and Newman's theories. European Journal of Personality, 16(5), 333-350. doi:10.1002/per.459 
Grandjean, D. (2005). The voices of wrath: Brain responses to angry prosody in meaningless speech. Nature Neuroscience, 8(2), 145.

Grossman, R. B., Bemis, R. H., Skwerer, D. P., \& Tager-Flusberg, H. (2010). Lexical and affective prosody in children with high functioning autism. Journal of Speech, Language, and Hearing Research, 53(3), 778. doi:10.1044/1092-4388(2009/08-0127)

Hansen, C. H., \& Hansen, R. D. (1988). Finding the face int he crowd: An anger superiority effect. Journal of Personality and Social Psychology, 54(6), 917-924. doi:10.1037/0022-3514.54.6.917

Harms, M. B., Martin, A., \& Wallace, G. L. (2010). Facial emotion recognition in autism spectrum disorders: A review of behavioral and neuroimaging studies. Neuropsychology Review, 20(3), 290. doi:10.1007/s11065-010-9138-6

Hawk, S. T., van Kleef, G. A., Fischer, A. H., \& van der Schalk, J. (2009). "Worth a thousand words": Absolute and relative decoding of nonlinguistic affect vocalizations. Emotion, 9(3), 293-305. doi:10.1037/a0015178

Hoekstra, R. A., Bartels, M., Verweij, C. J. H., \& Boomsma, D. I. (2007). Heritability of autistic traits in the general population. Archives of Pediatrics and Adolescent Medicine, 161(4), 372-377. doi:10.1001/archpedi.161.4.372

Hurley, R. S., Losh, M., Parlier, M., Reznick, J. S., \& Piven, J. (2007). The Broad Autism Phenotype Questionnaire. Journal of Autism and Developmental Disorders, 37(9), 1679-1690. doi:10.1007/s10803-006-0299-3

Ingersoll, B. (2010). Broader autism phenotype and nonverbal sensitivity: Evidence for an association in the general population. Journal of Autism and Developmental Disorders, 40(5), 590-598. doi:10.1007/s10803-009-0907-0

Ingersoll, B., Hopwood, C. J., Wainer, A., \& Donnellan, M. B. (2011). A comparison of three self-report measures of the broader autism phenotype in a non-clinical sample. 
Journal of Autism and Developmental Disorders, 41(12), 1646-1657. doi:10.1007/s10803-011-1192-2

Isomura, T., Ogawa, S., Yamada, S., \& Shibasaki, M. (2014). Preliminary evidence that different mechanisms underlie the anger superiority effect in children with and without Autism Spectrum Disorders. Frontiers in Psychology, 5, 1-8. doi:10.3389/fpsyg.2014.00461

Jamil, R., Gragg, M. N., \& DePape, A.-M. (2017). The broad autism phenotype: Implications for empathy and friendships in emerging adults. Personality and Individual Differences, 111, 199-204. doi:10.1016/j.paid.2017.02.020

Jobe, L. E., \& White, S. W. (2007). Loneliness, social relationships, and a broader autism phenotype in college students. Personality and Individual Differences, 42, 1479-1489. doi:10.1016/j.paid.2006.10.021

Johnstone, T., \& Scherer, K. R. (2000). Vocal communication of emotion. In M. Lewis \& J. Haviland (Eds.), The handbook of emotions (pp. 220-235). New York: Guilford.

Juslin, P. N., \& Laukka, P. (2001). Impact of intended emotion antensity on cue utilization and decoding accuracy in vocal expression of emotion. Emotion, 1(4), 381-412. doi:10.1037//1528-3542.1.4.381

Kadak, M. T., Demirel, O. F., Yavuz, M., \& Demir, T. (2014). Recognition of emotional facial expressions and broad autism phenotype in parents of children diagnosed with autistic spectrum disorder. Comprehensive Psychiatry, 55, 1146-1151.

Kiesler. (1983). The 1982 Interpersonal Circle: A taxonomy for complementarity in human transactions. Psychological Review, 90(3), 185-214. doi:10.1037/0033-295X.90.3.185

Kunihira, Y., Senju, A., Dairoku, H., Wakabayashi, A., \& Hasegawa, T. (2006). 'Autistic' Traits in Non-Autistic Japanese Populations: Relationships with Personality Traits 
and Cognitive Ability. Journal of Autism and Developmental Disorders, 36(4), 553566.

Lamport, D., \& Turner, L. A. (2014). Romantic attachment, empathy, and the broader autism phenotype among college students. The Journal of Genetic Psychology, 175(3-4), 202-213. doi:10.1080/00221325.2013.856838

Laukka, P., Juslin, P. N., \& Bresin, R. (2005). A dimensional approach to vocal expression of emotion. Cognition and Emotion, 19(5), 633. doi:10.1080/02699930441000445

Le Couteur, A., Bailey, A., Goode, S., Pickles, A., Gottesman, I., Robertson, S., \& Rutter, M. (1996). A broader phenotype of autism: The clinical spectrum in twins. The Journal of Child Psychology and Psychiatry, 37(7), 785-801. doi:10.1111/j.14697610.1996.tb01475.x

Leary, T. (1957). Interpersonal diagnosis of personality. New York: Ronald Press.

Lindner, J. L., \& Rosen, L. A. (2006). Decoding of emoiton through facial expresison, prosody and verbal content in children and adolescents with Apserger's syndrome. Jounral of Autism and Developmental Disorders, 36(6), 769. doi:10.1007/s10803006-0105-2

Lommen, M. J. J., Engelhard, I. M., \& van den Hout, M. A. (2010). Neuroticism and avoidance of ambiguous stimuli: Better safe than sorry? Personality and Individual Differences, 49, 1001-1006. doi:10.1016/j.paid.2010.08.012

Losh, M., Childress, D., Lam, K., \& Piven, J. (2008). Defining key features of the broad autism phenotype: A comparison across parents of multiple- and single-incidence autism families. American Journal of Medical Genetics. Part B: Neuropsychiatric Genetics, 147B, 424-433. doi:10.1002/ajmg.b.30612

McCrae, R. R., \& John, O. P. (1992). An Introduction to the Five-Factor Model and Its Applications. Journal of Personality. doi:10.1111/j.1467-6494.1992.tb00970.x 
Öhman, A., Lundqvist, D., \& Esteves, F. (2001). The face in the crowd revisited: A threat advantage with schematic stimuli. Journal of Personality and Social Psychology, 80(3), 381-396. doi:10.1037/0022-3514.80.3.381

Palermo, M. T., Pasqualetti, P., Barbati, G., Intelligente, F., \& Rossini, P. M. (2006). Recognition of schematic facial displays of emotion in parents of children with autism. Autism, 10(4), 353-364. doi:10.1177/1362361306064431

Pell, M. D., Paulmann, S., Dara, C., Alasseri, A., \& Kotz, S. A. (2008). Factors in the recognition of vocally expressed emotions: A comparison of four languages. Journal of Phonetics, 37(4), 417-435. doi:10.1016/j.wocn.2009.07.005

Philip, R. C. M., Whalley, H. C., Stanfield, A. C., Sprengelmeyer, R., Santos, I. M., Young, A. W., ... Hall, J. (2010). Deficits in facial, body movement and vocal emotional processing in autism spectrum disorders. Psychological Medicine, 40(11), 1919-1929. doi:10.1017/S0033291709992364

Pickles, A., Starr, E., Kazak, S., Bolton, P., Papanikolauo, K., Bailey, A., . . Rutter, M. (2000). Variable expression of the autism broader phenotype: findings from extended pedigrees. The Journal of Child Psychology and Psychiatry, 41(4), 491-502. doi:10.1111/1469-7610.00634

Poljac, E., Poljac, E., \& Wagemans, J. (2012). Reduced accuracy and sensitivity in the perception of emotional facial expressions in individuals with high autism spectrum traits. Autism, 17(6), 668-680. doi:10.1177/1362361312455703

Rosset, D., Santos, A., Da Fonseca, D., Rondan, C., Poinso, F., \& Deruelle, C. (2011). More than just another face in the crowd: Evidence for an angry superiority effect in children with and without autism. Research in Autism Spectrum Disorders, 5(2), 949956. doi:10.1016/j.rasd.2010.11.005 
Rutherford, M. D., Baron-Cohen, S., \& Wheelwright, S. (2002). Reading the mind in the voice: a study with normal adults and adults with Asperger syndrome and high functioning autism. Journal of Autism and Developmental Disorders, 32, 189-194. doi:10.1023/A:1015497629971

Sander, D., Grandjean, D., Pourtois, G., Schwartz, S., Seghier, M. L., Scherer, K. R., \& Vuilleumier, P. (2005). Emotion and attention interactions in social cognition: Brain regions involved in processing anger prosody. NeuroImage, 28, 848-858. doi:10.1016/j.neuroimage.2005.06.023

Sasson, N. J., Lam, K. S. L., Childress, D., Parlier, M., Daniels, J. L., \& Piven, J. (2013). The Broad Autism Phenotype Questionnaire: Prevalence and diagnostic classification. Autism Research, 6, 134-143. doi:10.1002/aur.1272

Sasson, N. J., Lam, K. S. L., Parlier, M., Daniels, J. L., \& Piven, J. (2013). Autism and the broad autism phenotype: Familial patterns and intergenerational transmission. Journal of Neurodevelopmental Disorders, 5(1), 11-18. doi:10.1186/1866-1955-5-11

Sasson, N. J., Nowlin, R. B., \& Pinkham, A. E. (2013). Social cognition, social skill, and the broad autism phenotype. Autism, 17(6), 655-667. doi:10.1177/1362361312455704

Scherer, K. R. (2003). Vocal communication of emotion: A review of research paradigms. Speech Communication, 40, 227-256. doi:10.1016/S0167-6393(02)00084-5

Schröder, M. (2003). Experimental study of affect bursts. Speech Communication, 40(1-2), 99-116. doi:10.1016/S0167-6393(02)00078-X

Schultz, P. W., Stone, W. F., \& Christie, R. (1997). Authoritarianism and mental rigidity: The Einstellung problem revisited. Personality and Social Psychology Bulletin, 23(1), 3-9.

Simon-Thomas, E. R., Keltner, D. J., Sauter, D., Sinicropi-Yao, L., \& Abramson, A. (2009). The voice conveys specific emotions: Evidence from vocal burst displays. Emotion, 9(6), 838-846. doi:10.1037/a0017810 
Sokal, R. R., \& Rohlf, F. J. (1995). Biometry: The principles and practice of statistics in biological research (3rd ed.). New York: W H Freeman.

Sucksmith, E., Roth, I., \& Hoekstra, R. A. (2011). Autistic traits below the clinical threshold: Re-examining the broader autism phenotype in the 21st century. Neuropsychology Review, 21(4), 360-389. doi:10.1007/s11065-011-9183-9

Tajmirriyahi, M., Nejati, V., Pouretemad, H., \& Sepehr, R. M. (2013). Reading the mind in the face and voice in parents of children with autism spectrum disorders. Research in Autism Spectrum Disorders, 7(12), 1543-1550. doi:10.1016/j.rasd.2013.08.007

Tong, E. M. W. (2010). Personality Influences in Appraisal-Emotion Relationships: The Role of Neuroticism. Journal of Personality, 78(2), 393-418. doi:10.1111/j.14676494.2010.00620.x

Tracey, T. J. G. (2005). Interpersonal rigidty and complementarity. Journal of Research in Personality, 39(6), 592-614. doi:10.1016/j.jrp.2004.12.001

Virkud, Y. V., Todd, R. D., Abbacchi, A. M., Zhang, Y., \& Constantino, J. N. (2009). Familial aggregation of quantitative autistic traits in multiplex versus simplex autism. American Journal of Medical Genetics. Part B: Neuropyschiatric Genetics, 150B(3), 328-334. doi:10.1002/ajmg.b.30810

Wainer, A. L., Ingersoll, B. R., \& Hopwood, C. J. (2011). The structure and nature of the broader autism phenotype in a non-clinical sample. Journal of Psychopathology and Behavioral Assessment, 33, 459-469. doi:10.1007/s10862-011-9259-0

Wakabayashi, A., Baron-Cohen, S., \& Wheelwright, S. (2006). Are autistic traits an independent personality dimension? A study of the Autism-Spectrum Quotient (AQ) and the NEO-PI-R. Personality and Individual Differences, 41(5), 873-883. doi:10.1016/j.paid.2006.04.003 
Walker-Andrews, A. S. (1997). Infants' Perception of Expressive Behaviors: Differentiation of Multimodal Information. Psychological Bulletin, 121(3), 437-456. doi:10.1037/0033-2909.121.3.437

Watson, D., \& Clark, L. A. (1984). Negative affectivity: The disposition to experience aversive emotional states. Psychological Bulletin, 96, 465-490. doi:10.1037/00332909.96.3.465

Wechsler, D. (1999). Wechsler Abbreviated Scale of Intelligence (WASI). San Antonio, TX: The Psychological Corporation.

Zeifman, D. M. (2001). An ethological analysis of human infant crying: Answering Tinbergen's four questions. Developmental psychobiology, 39(4), 265. doi:10.1002/dev.1005 
Figure Captions

Figure 1. Graphic interface of a trial from the online Emotion Recognition Task. In this trial, the emotion category of surprise and intensity rating of 4 were selected in response to a MAV stimulus. The location of each basic emotion category was randomized around the neutral category after every seven trials

Figure 2. Mean recognition accuracy for each emotion category from the present study $(N=$ $61)$ and Belin et al.'s (2008) original study on the validation of MAV stimuli $(N=30)$. In Belin et al. (2008), means were reported with standard errors, which were converted to 95\% confidence intervals represented by error bars in this figure

Figure 3. Mean intensity ratings for vocal affect bursts representing the six basic emotions. ( maximum score per emotion $=42$ points). Error bars represent $95 \%$ confidence intervals

Figure 4. Mean self-ratings of BAP features on scales of the Broad Autism Phenotype Questionnaire (BAPQ; maximum score per scale $=6$ points). Error bars represent $95 \%$ confidence intervals

Table 1 Characteristics of the sample $(N=61)$

\begin{tabular}{lr}
\hline Age & \\
Range & $18-70$ \\
$M$ & 30.56 \\
$S D$ & 11.34
\end{tabular}

Full-Scale IQ ${ }^{\mathrm{a}}$

Range 100-140 


\begin{tabular}{lc}
\hline$M$ & 119.15 \\
$S D$ & 8.97 \\
Educational Background & \\
Postgraduate (Master / PhD) & $60.7 \%$ \\
Undergraduate (Bachelor) & $37.7 \%$ \\
Year 12 & $1.6 \%$ \\
Nationality & \\
Australian & $47.5 \%$ \\
Asian & $36.0 \%$ \\
Other $^{\mathrm{b}}$ & $16.4 \%$ \\
\hline
\end{tabular}

${ }^{a}$ General intellectual functioning was assessed using the Wechsler Abbreviated Scale of Intelligence (WASI; Wechsler, 1999). ${ }^{\text {b }}$ Participants of various European, African, Middle Eastern, North American, and South American nationalities were also included. 
Table 2 Correlations between emotion recognition accuracy and Broad Autism Phenotype Questionnaire (BAPQ) scores

\begin{tabular}{lcccc}
\hline & \multicolumn{4}{c}{ BAPQ Scales } \\
\cline { 2 - 5 } Emotion Category & Total & Aloof & $\begin{array}{c}\text { Pragmatic } \\
\text { Language }\end{array}$ & Rigid \\
\hline Total & .078 & .053 & .011 & .124 \\
Anger & .214 & .215 & -.043 & $.315^{*}$ \\
Disgust & .040 & -.011 & .140 & -.014 \\
Fear & -.095 & -.026 & -.152 & -.071 \\
Happiness & -.044 & -.088 & -.104 & .084 \\
Sadness & -.113 & -.085 & -.038 & -.146 \\
Surprise & .065 & .035 & .076 & .056 \\
\hline
\end{tabular}

Note. Pearson's $r$ product-moment correlation coefficients are reported here.

$* p<.05$. 
Table 3 Correlations between emotion intensity ratings and Broad Autism Phenotype Questionnaire (BAPQ) scores

\begin{tabular}{|c|c|c|c|c|}
\hline \multirow[b]{3}{*}{ Emotion Category } & \multicolumn{4}{|c|}{ BAPQ Scales } \\
\hline & Total & Aloof & Pragmatic & Rigid \\
\hline & & & Language & \\
\hline Total & .033 & -.043 & -.023 & .154 \\
\hline Anger & .192 & .179 & -.027 & $.289^{*}$ \\
\hline Disgust & .087 & -.031 & .173 & .097 \\
\hline Fear & -.072 & -.064 & -.099 & -.013 \\
\hline Happiness & .049 & -.030 & -.055 & .204 \\
\hline Sadness & -.101 & -.154 & -.110 & .031 \\
\hline Surprise & .022 & -.096 & .070 & .000 \\
\hline
\end{tabular}

Note. Pearson's $r$ product-moment correlation coefficients are reported here.

$* p<.05$.

Figure 1 top

\section{WHAT IS THE EMOTION}

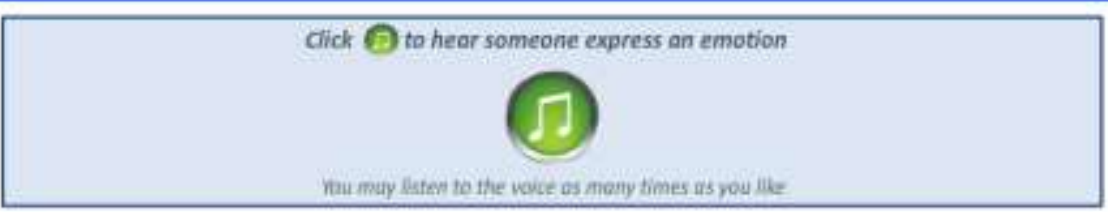

Think about the type of emotion expressed in their voice. Then, rate how strong the emotion sounds by clicking on a number in the line, from 1 (weak emotion) to 7 (strong emotion). If no emotion is expressed in their woice, then click on the circle in the middie.
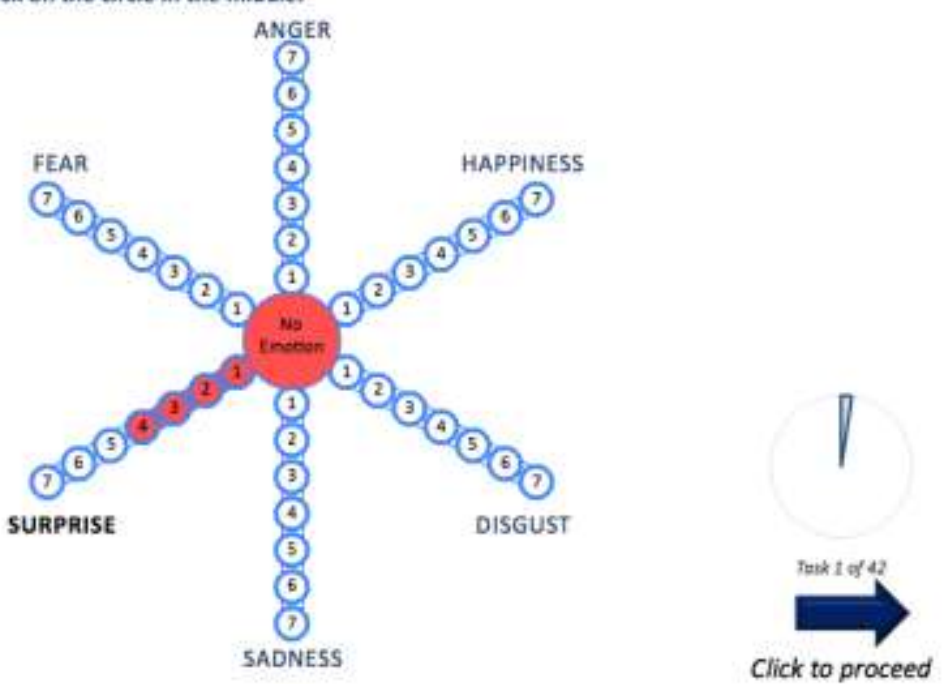
Figure 2 top

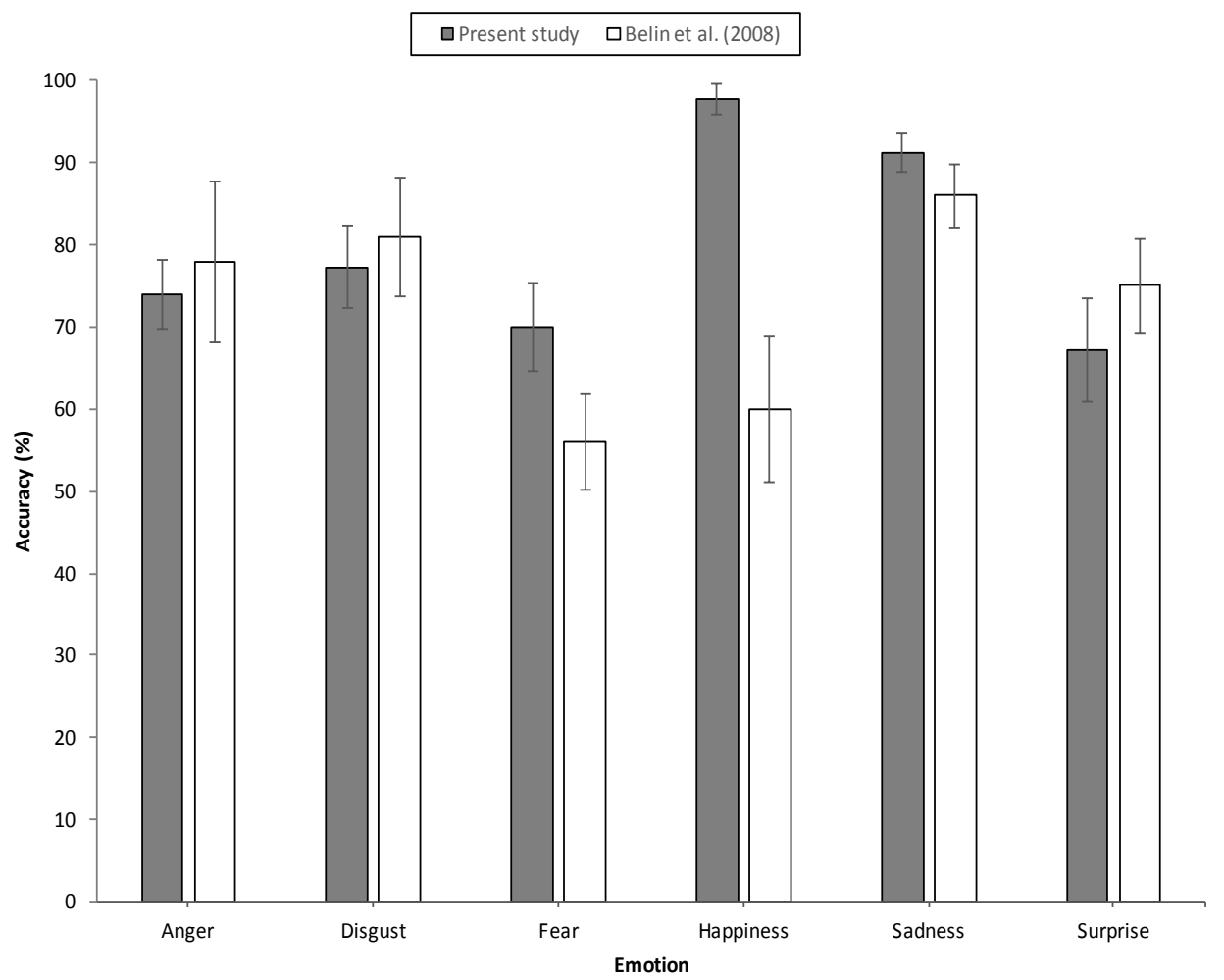

Figure 3 top 


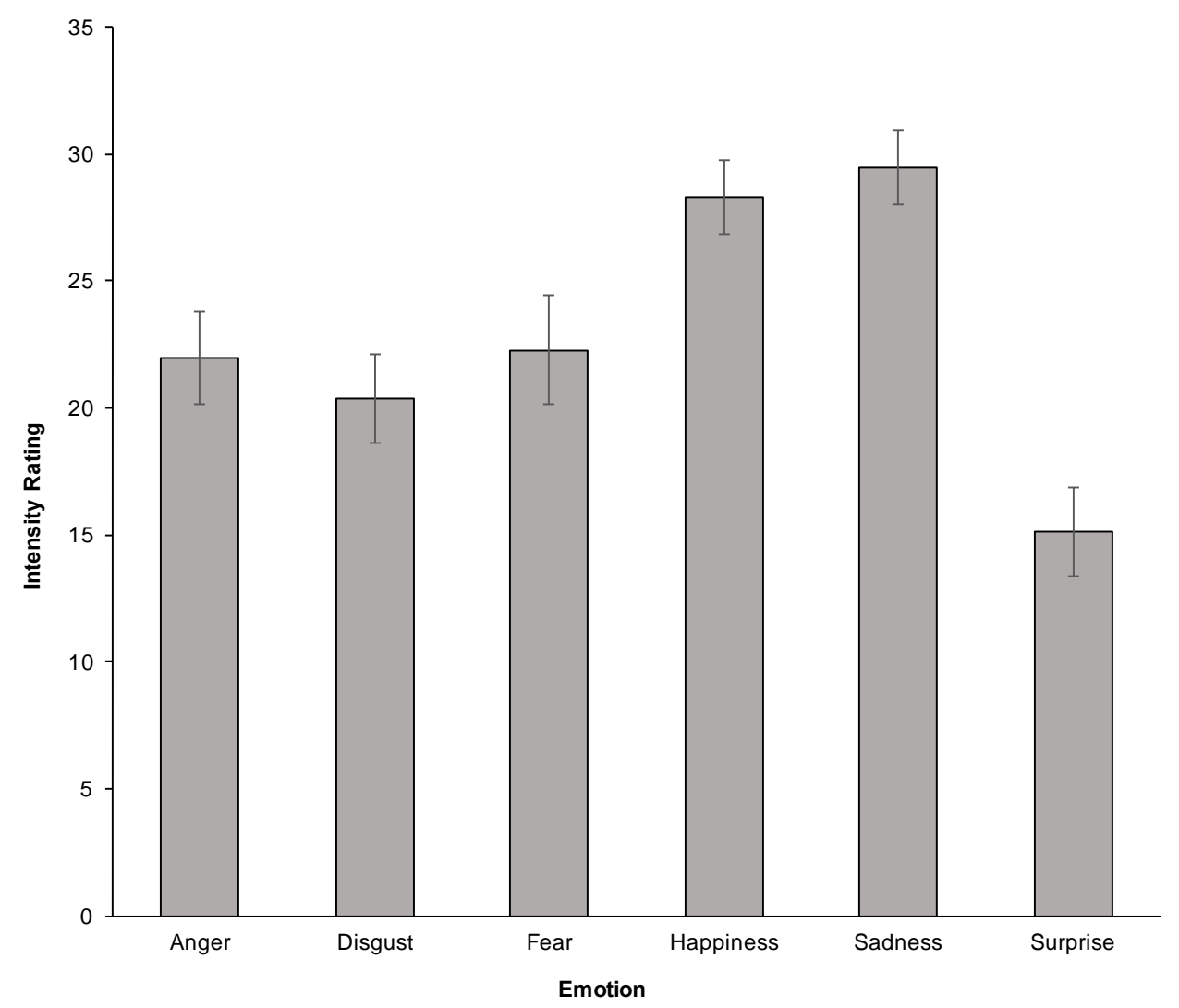

Figure 4 top 


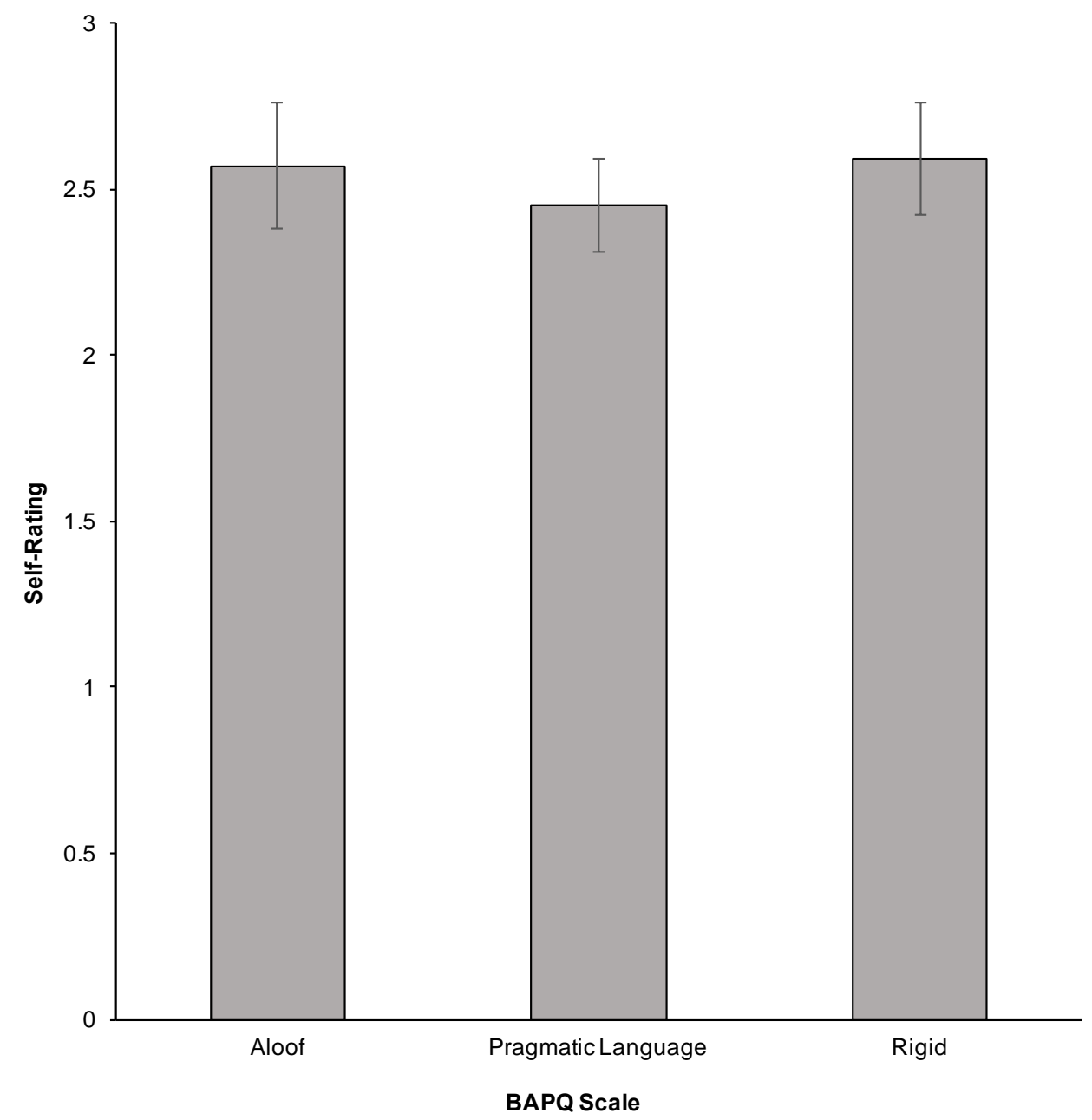




\section{University Library}

\section{- M M N E R VA A gateway to Melbourne's research publications}

Minerva Access is the Institutional Repository of The University of Melbourne

Author/s:

Yap, VMZ;McLachlan, NM;Scheffer, IE;Wilson, SJ

Title:

Enhanced Sensitivity to Angry Voices in People with Features of the Broader Autism Phenotype

Date:

2018-11-01

\section{Citation:}

Yap, V. M. Z., McLachlan, N. M., Scheffer, I. E. \& Wilson, S. J. (2018). Enhanced Sensitivity to Angry Voices in People with Features of the Broader Autism Phenotype. JOURNAL OF AUTISM AND DEVELOPMENTAL DISORDERS, 48 (11), pp.3899-3911. https:// doi.org/10.1007/s10803-018-3641-7.

Persistent Link:

http://hdl.handle.net/11343/283091 